\title{
Title: Evaluation of the Orthodontic Component of the Hypodontia Care Pathway
}

\section{Role of Authors:}

M. O'Keeffe: Responsible for conducting the research including patient recruitment, interviewing patients, analysis of results/content analysis and write up of the paper.

J.M. Collins: Responsible for study design, supervision of the research, critical revision of the paper and expert advice.

S.J. Cunningham: Responsible for study design; analysis of results/content analysis, supervision of the research, critical revision of the paper and expert advice.

*corresponding author - s.cunningham@ucl.ac.uk

Word count of abstract: 210

Word count of manuscript: 3568

Number of tables: 4 


\begin{abstract}
:
Introduction: This study evaluated patients' experiences of the Hypodontia Care Pathway at a large teaching hospital at key stages: specifically patient expectations/experience following the diagnosis of hypodontia and then patient satisfaction with the orthodontic care received and the outcome at the end of active orthodontic treatment. Methodology: In-depth, semi-structured interviews were conducted with twenty hypodontia patients following completion of orthodontic treatment but prior to any planned prosthodontic treatment commencing. Interviews were analysed qualitatively, using a framework approach. Results: The framework analysis identified four main themes: perceptions of treatment, impact of the original malocclusion and the treatment process, the care team and communication. The themes were then further divided into subthemes. Discussion: There was a large amount of positive feedback and the importance of the patient-clinician relationship was evident throughout. Where negative feedback was provided it mainly related to communication and areas were identified where change could take place in the future. Conclusions: Positive feedback was received and the importance of patient-clinician rapport was highlighted in all interviews. The main areas for improvement related to the importance of ensuring optimum communication, particularly with a cohort of patients who are often undergoing complex multidisciplinary treatment. Recommendations for the service have been made.
\end{abstract}

Keywords: Hypodontia, qualitative research, care pathway, patient satisfaction, impact 


\section{Introduction}

The prevalence of hypodontia in the permanent dentition is estimated to be $3.5-6 \%$ in the United Kingdom (Brook, 1974), with a 1.22 times more likely chance of hypodontia occurring in females than in males (Khalaf et al. 2014). The aetiology is complex, with genetics, the environment and developmental timing all believed to be factors which play a role (Cobourne, 2007; Fleischmannova et al., 2008).

Patients with hypodontia commonly present complaining of spacing between their teeth and poor dental aesthetics (Hobkirk et al., 1995). Missing anterior teeth are considered to be one of the most unattractive dental traits (Shaw, 1981). Individuals who have a less aesthetic dental appearance, such as conspicuous spacing, may also attract unfavourable social responses and become targets for nicknames and teasing from other children (Shaw et al., 1980; Seehra et al., 2011). Hypodontia has been proven to affect social and emotional well-being, although this does not appear to be related to the number of teeth missing (Kotecha et al., 2013).

Hypodontia is often treated by a multi-discplinary team approach, with input from orthodontics, paediatric dentistry, and prosthodontics. A multi-disciplinary approach meets the needs of the patients and their families by reducing the number of appointments required and by providing an opportunity for patients to meet all relevant specialties at the outset (Tams and Ashley, 2013), however, as yet there are no defined care pathways in place for hypodontia patients within the UK National Health Service. Ideally patients 
would make predictable transitions through each stage of the pathway and this would result in them receiving timely and optimal levels of care.

Part of the care pathway process also involves assessing outcomes and until relatively recently measuring orthodontic outcomes was primarily clinician led, using tools such as PAR scores or cephalometric outcomes. To date there are few tools in place to measure outcome from a patient perspective. Keles and Bos (2013) carried out a study in the Netherlands to identify specific factors that influenced patient satisfaction with orthodontic treatment and showed that the patient-doctor relationship was the most important factor contributing to patient satisfaction. Feldman (2014) carried out a similar study in Sweden and found that care and attention were the factors that showed the highest correlation with satisfaction. Neither of these studies investigated hypodontia specifically but it is likely that these findings are of importance to all orthodontic patients. Patients' perceptions of outcomes have been identified as a valuable source of information that can be used to improve services and Lord Darzi's report (2008) "High Quality of Care for All" highlighted the need for the success of a treatment intervention to include patient's views. A validated, hypodontia specific questionnaire has been designed to assess the impact of the condition on quality of life (QoL) and to investigate the effects of treatment (Akram et al., 2011, 2013) and this questionnaire is an important tool for the future. Whilst questionnaire based studies provide important findings, they do not always capture the detail which can be important when planning dental services, and this is where qualitative methods add to the overall picture and can be used to gain invaluable information regarding patients' perceptions. 


\begin{abstract}
$\underline{\text { Aim }}$
The aim of this study was therefore to use qualitative methods to evaluate and understand patients' experiences of the Hypodontia Care Pathway following completion of orthodontic treatment and prior to any necessary restorative treatment. Additionally to assess if patients were satisfied with the service and care provided through the pathway in a large teaching hospital in the UK.
\end{abstract}

\title{
Subjects and Methods
}

\section{$\underline{\text { Ethics }}$}

This study was granted favourable ethical opinion by the National Research and Ethics Service Committee West of Scotland (REC reference number 14/WS/1044).

\section{Study Group}

Participants were selected for interview using purposive sampling based on age, gender, ethnicity, the number of teeth missing and whether the missing teeth were to be prosthetically replaced or the spaces closed orthodontically. Patients who indicated their interest in being involved in the study were provided with a patient information leaflet (PIL), giving more detailed information and one of the research team (MO'K) discussed what would be involved. Patients who confirmed their willingness to be involved provided written consent. 


\section{Data Collection}

One member of the research team (MO'K) was chosen to carry out all of the interviews and interview training was provided. A topic guide was developed at the start of the study and was amended throughout the interview process. The topic guide was useful for directing the interviewer and ensuring all aspects were explored by acting as an aide memoir but was not followed strictly and, if new topics arose, the interviewer was free to explore these.

Patients were interviewed in a private side room within the Orthodontic Department of the Eastman Dental Hospital, UCLH Foundation Trust, and the interviews varied in length, with no time limit. The individual interviews ceased when patients had no further information to give. All interviews were recorded using a Dictaphone, anonymised and then transcribed in a Microsoft Word document.

\section{Data Analysis}

The interview transcripts were read and re-read until common themes were identified. Two members of the research team analysed the transcripts and discussed the findings until the themes and subthemes were agreed upon. The data from the transcribed interviews was then managed using a framework analysis. This is a matrix based analytic method, which was first developed by Ritchie and Spencer (1994) at the National Centre for Social Research and is widely used by qualitative researchers as it allows for the systematic management of data (Ritchie and Lewis, 2003). 
One spreadsheet in an Excel@ $\bigcirc$ workbook was dedicated to each theme and the columns represented subthemes, any non-verbal analytical comments were also added. The participants were then allocated a row each and relevant quotes from the transcripts were entered into the cells, along with the line numbers from transcripts.

\section{$\underline{\text { Results }}$}

Twenty patients were interviewed, six females and fourteen males, with ages ranging from 16 to 47 years old (average 21 years). Four main themes were identified from the interviews:

1. Perceptions of treatment

2. Impact of the original malocclusion and the treatment process

3. Care team

4. Communication

\section{Perceptions of treatment}

Patients reported being generally happy with the clinical care and service they received and commented on how good communication led to them feeling pleased with their understanding of the treatment process. The friendliness of the staff was mentioned in relation to overall care and the majority of patients commented that they were happy with the service, although a small number of suggestions for improvement were given. The duration of treatment was commented on by a number of patients who felt disappointed at 
the overall treatment times and felt it should have been quicker (Table 1).

\begin{tabular}{|l|l|}
\hline \multicolumn{1}{|c|}{ Subthemes } & \multicolumn{1}{|c|}{ Quotes } \\
\hline Satisfaction with clinical care & $\begin{array}{l}\text { I felt that I was looked after. I felt that every part of } \\
\text { the treatment was explained to me so I knew exactly } \\
\text { what was going to happen. (P1; 289) }\end{array}$ \\
\hline Satisfaction with the service & $\begin{array}{l}\text { All the dentists I had were really nice and friendly } \\
\mathbf{( P 6 ; 8 6 )}\end{array}$ \\
\hline Satisfaction with outcome & $\begin{array}{l}\text { I think they (the teeth) look incredible, I am so happy. } \\
\text { They look so good. } \mathbf{( P 2 0 ;} \text { 226) }\end{array}$ \\
\hline Suggestions for improvement & $\begin{array}{l}\text { I hay a normal looking mouth for the first time. } \\
\mathbf{( P 9} ; \mathbf{1 9 1})\end{array}$ \\
\hline
\end{tabular}

Table 1: Perceptions of treatment theme and subthemes

The patient number and line number from the transcript are in brackets after each quote.

Impact of the original malocclusion and the treatment process

The majority of the patients discussed that they did not like their teeth prior to treatment, with a large number confiding that they did not smile in photographs and were uncomfortable smiling generally prior to treatment. The appearance of retained primary 
teeth was commented on, with the focus on how they were shorter and darker than the permanent dentition. This was more of a concern when the primary teeth were retained anteriorly.

A number of patients then described how happy they were with their smile at the end of treatment and some also discussed feeling more confident when the retainers were in. Pontics on the retainers were highlighted as a positive aspect and the replacement of missing anterior teeth during fixed appliance treatment (with pontics on the archwire) was also seen as a favourable aspect of care (Table 2).

\begin{tabular}{|l|l|}
\hline \multicolumn{1}{|c|}{ Subthemes } & \multicolumn{1}{c|}{ Quotes } \\
Impact of $\quad$ original & I had a lot of gaps between my teeth and most of them \\
were still baby teeth. My two front teeth went towards \\
one side so they didn't look great. (P6; 123)
\end{tabular}




\begin{tabular}{|l|l|}
\hline Impact of retainers & $\begin{array}{l}\text { It made me feel better, it made me feel like I had a } \\
\text { full set of teeth. }(\mathbf{P 2} ; \mathbf{2 8 2})\end{array}$ \\
\hline
\end{tabular}

Table 2: Impact of the original malocclusion and the treatment process theme and subthemes

\section{Care Team}

A number of the participants had more than one clinician during the course of their treatment, however most understood why their clinician was changing and understood that their clinicians were on a training programme. A small number of patients commented on how they felt the transition difficult from one clinician to another, with one patient stating they "shy perhaps and a bit worried" (P2;174-176)

A large proportion of the participants were travelling long distances from home to attend their appointments and this had resulted in both financial costs and costs in terms of time off school or college. Some interviewees appeared confused regarding the role of their own general dental practitioner during treatment and a small number of patients did not attend their general dental practitioner for routine check-ups during the course of their fixed appliance treatment.

The majority of the participants attended the casualty/emergency service during the course of their orthodontic treatment. There was very positive feedback on this service, with all patients who attended reporting a good experience. Very few negatives were reported although occasional long waits were mentioned (Table 3). 


\begin{tabular}{|c|c|}
\hline Subthemes & Quotes \\
\hline Number of clinicians & $\begin{array}{l}\text { I wasn't really bothered but obviously there was a lot } \\
\text { of change, maybe more stability might have helped } \\
\text { but it wasn't a major thing. (P12;74-75) } \\
\text { "shy perhaps and a bit worried" (P2;174-176) - } \\
\text { regarding the transition between clinicians. }\end{array}$ \\
\hline Appointments & $\begin{array}{l}\text { Because I go to Uni and I don't live here she } \\
\text { definitely took that into consideration which was } \\
\text { good so I didn't miss Uni. (P10; 88-89) }\end{array}$ \\
\hline $\begin{array}{l}\text { Time off school/ college/work } \\
\text { Cost implications }\end{array}$ & $\begin{array}{l}\text { I had to keep coming down from uni if they broke so } \\
\text { it was costing me a lot of money. }(\mathbf{P} 17 ; \mathbf{1 4 0 )}\end{array}$ \\
\hline $\begin{array}{l}\text { Care of the appliance and } \\
\text { attendance for casualty } \\
\text { appointments }\end{array}$ & $\begin{array}{l}\text { The emergency department is brilliant because } \\
\text { whoever it was they knew what to do. } \\
\text { (P8; 81) }\end{array}$ \\
\hline $\begin{array}{l}\text { General care of teeth and } \\
\text { visits to the general dental } \\
\text { practitioner }\end{array}$ & $\begin{array}{l}\text { I see him (GDP) every six months, he asks how it is } \\
\text { going, is everything ok, have they said if I need to do } \\
\text { anything. (P9; 207) }\end{array}$ \\
\hline
\end{tabular}

Table 3: Care Team theme and subthemes 


\section{Communication}

Communication was a key theme discussed by all of the interviewees and patients talked about the importance of communication at all stages of their treatment. Most patients felt the options prior to treatment were explained well to them and showed a good understanding of the decision making process, although this stage had taken place some years previously so there could have been an element of recall bias. Patients mentioned how they valued being involved in decisions made during treatment and valued their clinician explaining what was happening. They also appreciated being involved in the overall decision making process. When patients were asked about the next stage of their treatment, the majority had a good understanding and were clear about what their future treatment involved. However, one area of concern in communication related to treatment duration. Some participants commented that the overall duration was as described at the start of treatment, however a small number of participants were unhappy with the overall length of treatment, feeling it was longer than they were prepared for (Table 4).

\begin{tabular}{|l|l|}
\hline \multicolumn{1}{|c|}{ Subthemes } & \multicolumn{1}{c|}{ Quotes } \\
\hline decision making & $\begin{array}{l}\text { They will do a bridge on this side because I don't have } \\
\text { enough bone at all on this side. }(\mathbf{P 8 ;} \mathbf{1 4 4})\end{array}$ \\
\hline During treatment & $\begin{array}{l}\text { I heard the consultant and the person who was actually doing } \\
\text { the treatment discussing it and they would then explain it to } \\
\text { me afterwards. (P8; 93-95) }\end{array}$ \\
\hline
\end{tabular}




\begin{tabular}{|c|c|}
\hline $\begin{array}{l}\text { Post treatment \& } \\
\text { future treatment }\end{array}$ & $\begin{array}{l}\text { I think I am going to get a bridge and once the tooth has been } \\
\text { put in I am going to get a retainer wire that goes around the } \\
\text { back of the teeth to hold them in place. (P7;134-137) }\end{array}$ \\
\hline Decision making & $\begin{array}{l}\text { You're pleased you are given choices, it's empowering yet } \\
\text { you're like but really I want you to do what's best, what's } \\
\text { going to give me the best result. So I think its important to } \\
\text { know your options, that there are options, but ultimately you } \\
\text { want the people who are the professionals to say yeah but } \\
\text { really we think this is what we should do. (P14;187) } \\
\text { My older sister and brother had it done so my parents thought } \\
\text { it would be simpler to have the same thing done, so my } \\
\text { parents made the decision really. (P7; 67-68) }\end{array}$ \\
\hline Treatment duration & $\begin{array}{l}\text { I think they said it would take two or three years and it was } \\
\text { two and a half years I had them on for, so yeah it was about } \\
\text { right. (P8; 86) } \\
\text { Being told it was going to be one and a half to two years and } \\
\text { then have them on for three years, its quite different. } \\
\text { (P5; 70-72) }\end{array}$ \\
\hline
\end{tabular}

Table 4: "Communication" theme and subthemes 


\section{Discussion}

This study highlights the strengths of having a specific care pathway in place for hypodontia patients but also identifies areas which can be improved. Purposive Sampling was carried out by constructing a sampling framework. This was undertaken to capture variations which may exist within the hypodontia population. Despite efforts to interview patients with a range of severities of hypodontia, a large number of the patients, 9 of the 20 interviewed, had severe hypodontia. This may be due to the nature of the patients referred to the hypodontia clinic at the Eastman Dental Hospital and is a limitation of the study as severe hypodontia is relatively rare in the general population, with a prevalence of $0.3 \%$ of the general population (Hobkirk and Brook, 1980).

One researcher carried out all of the interviews and this ensured consistency throughout the interview process. The interviews took place in a quiet side room away from the main clinic to allow for privacy and to create a relaxed environment. All participants were sixteen years old or older and therefore no parents or family members were in the room during the interviews.

Analysis of the interviews led to four main themes. The first theme identified related to overall perceptions of treatment and this was subdivided into perceptions of clinical care, service, and outcome. However patients focused mainly on the relationship with their clinician, many patients commented on the friendliness and kindness of their orthodontist and the clinician's attention to them as an individual. Other patients commented on how they felt good communication led to good clinical care. As previously mentioned, the 
studies by Keles and Bos (2013) and Feldman (2014) also concluded that the relationship between orthodontist and patient is key to a successful outcome and is equally as important as a technically successful outcome.

A large number of the patients discussed how they felt about the outcome of treatment and commented on the appearance of their teeth following debond and how this improved appearance led to increased self-confidence and the ability to smile more confidently. It is understandable how the closure of spaces orthodontically, or provision of retainers with pontics, leads to an improvement in dental appearance and impacts on confidence. These findings are also in agreement with McNair et al. (2006) who examined the perceptions of orthodontic patients following orthodontic treatment and concluded that one of the main benefits of orthodontic treatment was an improvement in appearance and selfconfidence.

The second theme related to the impact of the original malocclusion and the treatment process. Many of the patients discussed feeling self-conscious about the spaces in their dentition prior to treatment and a number of patients reported that they avoided smiling in social situations and in photographs due to this. A smaller number of interviewees were concerned that people noticed the spacing, with one patient reporting an incident when a stranger asked why they had missing teeth/spaces. The majority of these comments referred to visible anterior spacing and spaces posteriorly did not have such a high impact. This is similar to the findings of Anweigi et al. (2013) who found that those patients in their study who scored highest on the Oral Health Impact Profile (Slade and Spencer, 
1994) were those who had spacing both anteriorly and posteriorly and therefore had the poorest quality of life. This highlights the need to restore anterior spaces early where possible, particularly in adolescent patients when appearance is a priority and this may make the transition into adulthood easier. Interim restorations or pontics may be placed during this period, with definitive care provided at a later stage when conditions are more favourable.

A large number of patients commented positively on their fixed appliances, with many stating that they liked them and few said that the fixed appliances negatively affected their confidence. One patient stated that fixed appliances gave her confidence as she felt that she was getting something done about her teeth and moving forward in her treatment. However, some of the patients who commenced their fixed appliance treatment as adults felt more conscious of the appliances. Some reported that they felt it may have impacted on their ability to get a job, with one patient stating that they did not receive a job offer until after the fixed appliances were debonded. A large number of patients commented positively on their retainers, with most of the comments relating to improved aesthetics and an increase in self-confidence. Interestingly, few of the patients had pontics on the archwire during fixed appliance treatment and this may account for the sudden improvement in aesthetics when retainers with pontics were fitted. When asked how they felt when provided with retainers some patients described feeling like they had a "full set of teeth for the first time". This reflects on the psychological impact of having missing teeth and how patients finally felt they were "dentally normal" once they had the spaces restored with a prosthesis, even if it was only temporary. There is little evidence 
published on the psycho-social impact of the provision of retainers with pontics following debond of orthodontic appliances. Further research in this area would be very interesting, particularly with respect to the psychological and functional gain of fitting orthodontic retainers with pontics and the use of pontics on the archwire during fixed appliance treatment.

The third major theme related to the care team. In a large teaching hospital it is not surprising that some patients had more than one clinician during their treatment. The majority of patients understood why the clinicians were changing and had the transition explained to them; good communication skills facilitated the change of clinician for both patient and clinician. Again the friendliness and kindness of the clinicians was commented on and this reinforces how the doctor-patient relationship plays an important role in patient satisfaction.

A useful way of reducing any concerns regarding clinician transfer is for the outgoing clinician to introduce the new clinician to the patient prior to the change in care. This "meet and greet" may put the patient at ease and the clinicians can also discuss the treatment plan and progress of treatment.

Mainly positive comments were received when asking about convenience of appointments, with many reporting that clinicians were accommodating and recognised that some patients were travelling long distances. Regular appointments over a long course of treatment can be financially demanding and this was commented on by a 
number of patients. In addition to the financial cost of travelling to and from appointments, patients often need to take time off school or work, which can be therefore create indirect costs. It is important that these issues are discussed with patients prior to starting treatment.

A large number of patients commented positively on the 'walk-in' casualty service, with the speed of the treatment being the main positive factor discussed. The treatment times were found to be efficient and most patients understood what element of their appliance had broken and why.

The majority of interviewees attended their general dental practitioner (GDP) for routine examinations during their orthodontic treatment, however it is of concern that some did not appear to fully appreciate their GDP's role during treatment and did not attend for regular checks. It is therefore imperative to remind all patients of the importance of regular attendance at their GDP during orthodontic treatment. Regular dental check-ups are vital for any orthodontic patient but especially, hypodontia patients, when attempts are often made to retain primary teeth for as long as possible and, due to the lengthy treatment often involved, good dental care and oral hygiene are critical.

Communication was highlighted as one of the four major themes. Most patients felt the treatment options were explained well prior to treatment starting and showed a good understanding of what was explained. Witt and Bartsch (1996) discussed the need for special training in communication for dentists/orthodontists and communication has been 
shown to be effective only if the content is easy to understand and recall (Ley and Spelman, 1967). In the current study a large number of patients reported on the friendliness and kindness of their clinicians and specifically discussed good communication issues. This was also found by Nanda and Kierl (1992) who examined predictors of co-operation in orthodontic treatment and found that the orthodontist-patient relationship had the strongest association with patient compliance. Witt and Barsch (1996) examined the effects of information exchange and communication and also identified the doctor-patient relationship as the key to successful patient compliance during treatment.

A number of patients in the current study were teenagers at initial consultation and therefore recall of information from the initial consultation may differ from the adult patients. Some of the patients discussed how they were very young at the time of the initial consultation and did not understand, or were not aware of, the magnitude of the condition and treatment at that time. Therefore, responsibility at that stage lies with the parents to act in the best interests of their child, hence the need to communicate well with both patient and parents.

Patients also commented that they appreciated being given options and having an input into the decision making process. One interviewee described how they felt "empowered" by the decision making process and how it was important to know all of the options, but many also discussed how they felt they needed to be guided by the clinicians in their decision making. Younger patients also talked about their parents' input in the decision- 
making process and previous family experiences of dental procedures and orthodontics were reflected on prior to decision-making.

Most patients understood what the next stage of their treatment entailed, with the majority having information regarding their future care. Patients appeared to accurately recall the plans for the next phase in their treatment and this may be due to repetition during the course of their orthodontic treatment, with regular updates and conversations regarding future plans.

As previously mentioned some participants felt treatment was prolonged and not what they were prepared for and this is a concern. O'Connor (2000) carried out a study in Ireland where 146 consecutive orthodontic patients completed questionnaires encouraging them to give opinions on their treatment. One of the main recommendations was the need for more accurate estimates of treatment duration. A study of conventional orthodontic treatment by McNair et al. (2006) also found that patients felt that more accurate estimates of treatment time would be a means of improving the service. This emphasises the importance of clinicians being clear regarding the overall length of treatment and to give accurate estimates, whilst highlighting the unpredictability of treatment times especially in multi-disciplinary treatments. Long courses of treatment can in turn have higher financial implications due to a combination of travel costs and time off from work. Accurate estimations of treatment duration would allow patients to prepare for this in advance. 
The multi-faceted impact of hypodontia is often overlooked and this study has also reinforced the need to be aware of the psycho-social aspects of the condition. By assessing care pathways using qualitative research methods, invaluable data can be generated which provides an insight into the multi-disciplinary journey of the hypodontia patient.

\section{Conclusion}

This study highlights the impacts associated with hypodontia and orthodontic treatment for this condition. A number of recommendations were made regarding treatment. Some of these recommendations included practical measures (for example, use of anterior pontics on the archwires wherever possible) but the majority related to communication. Although good communication was identified as a key strength of the service, methods of enhancing this were also considered.

- Running a communication skills seminar annually for all departmental staff.

- Developing a patient information leaflet, specific to the Eastman Dental Hospital, depicting the typical hypodontia care pathway and including photographs/explanations of appliances and procedures and photographs of key members of the hypodontia team.

- Introducing a "meet and greet" session when changing clinician in complex hypodontia cases.

- Clearly communicating the long duration of treatment for hypodontia patients, especially where restorative treatment is also involved. The importance of ensuring patients attend their general dental practitioner regularly during these 
extended treatments should also be reiterated.

\title{
Role of Authors:
}

M. O'Keeffe: Responsible for conducting the research including patient recruitment, interviewing patients, analysis of results/content analysis and write up of the paper

J.M. Collins: Responsible for study design, supervision of the research, critical revision of the paper and expert advice

S.J. Cunningham: Responsible for study design; analysis of results/content analysis, supervision of the research, critical revision of the paper and expert advice.

\section{References}

\author{
Akram AJ, Jerreat AS, Woodford J, Sandy JR, Ireland AJ. (2011) \\ Development of a condition-specific measure to assess quality of life in patients with \\ hypodontia \\ Orthodontics and Craniofacial Research. 14(3): 160-167
}

\begin{abstract}
Akram AJ, Ireland AJ, Postlethwaite KC, Sandy JR, Jerreat AS. (2013)
Assessment of a condition-specific quality-of-life measure for patients with developmentally absent teeth: validity and reliability testing Orthodontics and Craniofacial Research. 16(4): 193-201
\end{abstract}


Anweigi L, Allen PF, Ziada H. (2013)

The use of the Oral Health Impact Profile to measure the impact of mild, moderate and severe hypodontia on oral health-related quality of life in young adults

Journal of Oral Rehabilitation. 40(8): 603-608

\section{Brook AH. (1974)}

Dental anomalies of number, form and size: their prevalence in British schoolchildren.

Journal of the International Association of Dentistry for Children. 5: 37-53.

\section{Cobourne MT. (2007)}

Familial human hypodontia - is it all in the genes?

British Dental Journal. 203: 203-208.

Darzi A. (2008)

High quality care for all: NHS Next Stage Review Department of Health, England

\section{Feldmann I. (2014)}

Satisfaction with orthodontic treatment outcome.

The Angle Orthodontist. 84(4): 581-587.

Fleischmannova J, Matalova E, Tucker AS, Sharpe PT. (2008) 
Mouse models of tooth abnormalities.

European Journal of Oral Sciences. 116: 1-10.

Goodman JR, Jones SP, Hobkirk JA, King PA. (1994)

Hypodontia: 1. Clinical features and the management of mild to moderate hypodontia.

Dental Update. 21: 381-384.

Hess TM, Tate CS. (1991)

Adult age differences in explanations and memory for behavioural information.

Psychology and Aging 6: 86-92

Hobkirk JA, King PA, Goodman JR, Jones SP. (1995)

Hypodontia: 2. The management of severe hypodontia.

Dental Update. 22: 8-11.

Keles F, Bos A. (2013)

Satisfaction with orthodontic treatment.

The Angle Orthodontist. 83(3): 507-511.

Kessels R. (2003)

Patients' memory for medical information

Journal of the Royal Society of Medicine 96: 219-222. 
Khalaf K, Miskelly J, Voge E, Macfarlane TV. (2014)

Prevalence of hypodontia and associated factors: a systematic review and meta-analysis.

Journal of Orthodontics._41(4):299-316.

Kotecha, S., Turner, P. J., Dietrich, T., \& Dhopatkar, A. (2013)

The impact of tooth agenesis on oral health-related quality of life in children.

Journal of Orthodontics. 40(2): 122-129.

Langlois JH, Kalakanis L, Rubenstein AJ, Larson A, Hallam M, Smoot M. (2000)

Maxims or myths of beauty? A meta-analytic and theoretical review.

Psychological Bulletin. 126(3): 390-423

Ley P, Spelman M.(1967)

Communicating with the Patient.

London: Staple Press.

McNair A, Gardiner P, Sandy JR, Williams AC. (2006)

A qualitative study to develop a tool to examine patients' perceptions of NHS orthodontic treatment.

Journal of Orthodontics. 33(2): 97-106

Nanda RS, Kierl MJ (1992) 
Prediction of co-operation in orthodontic treatment.

American Journal of Orthodontics and Dentofacial Orthopedics 102: 15-21

\section{O’Connor PJ. (2000)}

Patients' perceptions before, during, and after orthodontic treatment.

Journal of Clinical Orthodontics. 34: 591-592.

Ritchie J, Spencer L, Bryman A, Burgess RG. (1994)

Analysing qualitative data.

London: Routledge 3.

Ritchie, J, Lewis, J. (2003)

Qualitative research practice: A guide for social science students and researchers.

1st Ed. SAGE Publications Limited. Pages 24 -29; 220-237

Seehra J, Fleming PS, Newton T, DiBiase AT. (2011)

Bullying in orthodontic patients and its relationship to malocclusion, self-esteem and oral health-related quality of life

Journal of Orthodontics. 38(4): 247-256

\section{Shaw WC, Addy M, Ray C. (1980)}

Dental and social effects of malocclusion and effectiveness of orthodontic treatment: a review 
Community Dentistry and Oral Epidemiology. 8(1) : 36-45

\section{Shaw WC. (1981)}

The influence of children's dentofacial appearance on their social attractiveness as judged by peers and lay adults

Journal of Orthodontics. 79(4): 399-415

\section{Slade GD, Spencer AJ. (1994)}

Development and evaluation of the Oral Health Impact Profile. Community Dental Health. 11(1): 3-11

Tams C, Ashley M. (2013)

Improving patient experience in a multi-disciplinary clinic: clinical efficiency and patient satisfaction of 400 patients attending the Manchester Hypodontia Clinic.

British Dental Journal. 214(5): E11-E11

Witt E, Bartsch A. (1996)

Effects of information-giving and communication during orthodontic consultation and treatment. Part 3: Optimized orthodontist-patient communication.

Journal of Orofacial Orthopedics. 57(3): 154-167. 